\title{
Agrupamento de colheitas de tomate e estimativas do tamanho de par- cela em cultivo protegido
}

\author{
Alessandro Dal'Col Lúcio; Ricardo H Carpes; Lindolfo Storck; Bernardo Zanardo; Marcos Toebe; Odair \\ José Puhl; Jorge Roque A dos Santos \\ UFSM-Depto. Fitotecnia, CCR, Camobí, 97105-900 Santa Maria-RS; adlucio@smail.ufsm.br (autor correspondente); rh.carpes@bol. \\ com.br; lindolfo@smail.ufsm.br; berzanardo@gmail.com; marcostoebe@mail.ufsm.br; odapuhl@gmail.com; jorgeroquealves@yahoo. \\ com.br
}

\section{RESUMO}

Para verificar qual a interferência da variabilidade das estimativas dos valores usados para determinação do tamanho de parcela com simulações de diferentes números de plantas por parcela e agrupamentos de colheitas, realizou-se um trabalho com três experimentos de tomate no Departamento de Fitotecnia da UFSM. Em dois experimentos foram utilizados túneis plásticos, com irrigação por gotejamento, camalhões com mulching preto de PEDB, três linhas de cultivo, 24 plantas por linha, espaçamento entre plantas $0,8 \mathrm{~m}$ e entre filas $1,2 \mathrm{~m}$ e uma estufa plástica no terceiro, essa com dimensões de $24 \mathrm{~m}$ de comprimento por $10 \mathrm{~m}$ de largura em oito linhas de cultivo. Todos os tratos culturais foram realizados com base na recomendação para a cultura (Filgueira, 2000), sendo aplicados concomitantemente nos três experimentos. A seqüência de duas, três e quatro plantas na linha de cultivo formou as parcelas de duas, três e quatro unidades básicas de tamanho. Foram realizadas análises considerando-se apenas colheitas individuais e colheitas agrupadas. Foram estimados a variância, o coeficiente de variação e o índice de informação relativa. Aplicou-se o teste de Bartlett entre as variâncias das colheitas individuais e agrupadas em cada tamanho de parcela simulado para verificar sua homogeneidade. As variâncias, entre as colheitas agrupadas, são homogêneas a partir do agrupamento de seis colheitas. Há acréscimos nas estimativas da variância da fitomassa de frutos com o aumento do tamanho da parcela e/ou o número de colheitas agrupadas. Parcelas de quatro plantas na linha de cultivo, combinados com agrupamento de seis em seis colheitas reduzem a variabilidade.

Palavras-chave: Lycopersicum esculentum L., precisão experimental, olericultura, ambiente protegido, tamanho de parcela.

\section{ABSTRACT \\ Grouping in tomato harvests and estimates of the plot size in protected environment}

The variability interference was verified on the estimates of the values used to determine the plot size with simulations of different numbers of plants per plot and groupings of harvests. Three experiments were performed with the tomato crop in the winter/ spring of 2007, accomplished in Santa Maria, Rio Grande do Sul State, Brazil. In two of the experiments we used a plastic tunnel, with drip irrigation, black mulching of PEDB, three lines with 24 plants per row, spacing between plants of $0.8 \mathrm{~m}$ and between rows of 1.2 $\mathrm{m}$ and a plastic greenhouse in the third experiment, $24 \mathrm{~m}$ long and $10 \mathrm{~m}$ wide in eight cultivation rows. Cultural practices were carried out as recommended for the culture, being applied concurrently in all experiments. The sequence of two, three and four plants in the crop line formed the plots of two, three and four UB of size. Analyses were accomplished considering only individual and combined harvests. For each one of the three experiments, the estimates of the variance, the variation coefficient and the index relative information had been obtained. The Bartlett test was applied among the variances of the individual and combined harvests in each size of simulated plot to verify the homogeneity among the variances. The variances, among the grouped harvests were homogeneous, considering the grouping of six harvests. There were some increases in the estimates of the average and in the variance of the fitomass of fruits when the size of the plot and/or the number of grouped harvests was increased. The arrangement of four plants in the cultivation rows, combined with grouping of six in six crops reduce the variability.

Keywords: Lycopersicum esculentum L., experimental precision, horticulture, protected environment, plot size.

(Recebido para publicação em 2 de julho de 2009; aceito em 28 de maio de 2010) (Received on July 2, 2009; accepted on May 28, 2010)

E 2006 a produção total de hortaliças foi de 17,26 milhões de toneladas, ocupando uma área cultivada de 785,2 mil ha, de acordo com Camargo Filho \& Camargo (2008). Do valor total da produção estimado em $\mathrm{R} \$ 11,4$ milhões, apenas seis hortaliças (tomate, batata, melancia, cebola, cenoura e batata-doce) respondem por mais de $64 \%$ do volume total produzido, com a cultura do tomate ocupando a segunda posição (IBGE, 2008).

As hortaliças são produzidas no
Brasil, predominantemente, pelo sistema de cultivo convencional, mas, nos últimos anos, tem se verificado um significativo crescimento de cultivos em ambiente protegido, principalmente pela maximização da produção, obtenção de produtos de alta qualidade, maior precocidade, melhor controle de pragas e doenças e economia da água de irrigação (Cermeño, 1990). Este sistema, também permitiu o cultivo com hortaliças fora da época preferencial e em lugares onde as condições locais são limitantes (Lorentz et al., 2004), influenciando diretamente a produção de hortaliças no país que nos últimos dez anos, segundo IBGE (2008), aumentou $33 \%$ enquanto a área foi reduzida em $5 \%$ e a produtividade incrementada em $38 \%$.

No planejamento experimental as definições do tamanho e forma de parcela, número de repetições, tamanho da amostra, delineamento experimental, entre outras características, são diretamente influenciadas pela variabilidade inerente ao experimento (Steel et al., 
1997; Storck et al., 2006). Esta variabilidade, conforme Storck et al. (2006), também interfere no momento da análise estatística dos resultados, inflacionando a estimativa do erro experimental, levando o pesquisador a interpretações e conclusões com baixa precisão experimental e confiabilidade.

Em cultivos de olerícolas em ambiente protegido, como estufas e túneis plásticos, fatores como a proximidade das linhas de cultivo com as laterais da estufa, a presença ou ausência de frutos aptos a serem colhidos em determinada colheita, as múltiplas colheitas realizadas para algumas culturas, o manejo cultural mais intensivo são, conforme Lorentz et al. (2005) e Lúcio et al. (2008), algumas fontes de variabilidade naquele sistema de cultivo que devem ser controlados durante a execução de experimentos nessas condições, evitando assim inflacionar a variabilidade já existente nessas condições de cultivo.

Trabalhando no sentido de identificar as interferências das fontes de variabilidade, presentes no sistema de cultivo em ambiente protegido, sob as diversas estimativas no planejamento de experimentos, autores como Lopes et al. (1998), Marodin et al. (2000), Souza et al. (2002), Mello et al. (2004), Lúcio et al. (2004), Lorentz et al. (2005), Lúcio et al. (2006), Feijó et al. (2008), Lúcio et al. (2008) e Carpes et al. (2008) apontaram que existe variabilidade significativa entre as linhas de cultivo e entre colheitas realizadas, independente da cultura olerícola avaliada, e que essa variabilidade altera de forma significativa as estimativas dos tamanhos de amostra, tipo de amostragem, tamanho e forma de parcela, delineamento experimental e número de colheitas suficientes para melhor discriminação entre os tratamentos estudados.

Com essas características inerentes ao sistema de cultivo de olerícolas em ambiente protegido, há então a necessidade de caracterizar a fonte de variabilidade mais proeminente e planejar o experimento de tal forma mantê-la sob controle. Como várias culturas olerícolas, como no caso do tomate, os frutos são colhidos em diferentes períodos, algumas plantas podem não apresentar frutos aptos a serem colhidos, ficando assim com uma produção nula naquele momento específico. Essa produção nula gera a presença, dentro do banco de dados, de várias observações com valores nulos, inflacionando a variabilidade já existente no experimento. Uma das hipóteses para minimizar os efeitos desse problema é trabalhar com parcelas com um número maior de plantas e/ou agrupar colheitas sucessivas, favorecendo a redução de parcelas com valores nulos de produção em colheitas específicas e, desta forma, reduzindo a variabilidade entre linhas de cultivo, entre parcelas dentro da mesma linha e entre colheitas realizadas.

O presente trabalho teve como objetivo estudar a variabilidade das estimativas usadas para determinar o tamanho de parcela com simulações de diferentes números de plantas por parcela e agrupamentos de colheitas em cultivo protegido.

\section{MATERIAL E MÉTODOS}

Os experimentos com a cultura do tomate híbrido Empire foram conduzidos na estação inverno/primavera (I/P) de 2007, no Departamento de Fitotecnia da Universidade Federal de Santa Maria, com latitude: $29^{\circ} 43$ 'S, longitude: $53^{\circ} 43$ 'W e altitude $95 \mathrm{~m}$, sendo o clima classificado como Cfa (subtropical úmido sem estação seca definida e com verões quentes) e o solo classificado como Argissolo Vermelho distrófico arênico (Embrapa, 1999).

Dois experimentos foram realizados em túneis plásticos com dimensões de 3,5 m de altura na parte central, $25 \mathrm{~m}$ de comprimento por $4 \mathrm{~m}$ de largura, com área útil de 19,2 $\mathrm{m}$ de comprimento e 3,6 m de largura, coberto com filme de Polietileno de Baixa Densidade (PEBD) de 200 micras, utilizando irrigação por gotejamento, camalhões com mulching preto de PEDB de 35 micras de espessura com $0,15 \mathrm{~m}$ de altura e $0,4 \mathrm{~m}$ de largura, três linhas de cultivo, 24 plantas por linha, espaçamento entre plantas de $0,8 \mathrm{~m}$ e entre linhas de cultivo $1,2 \mathrm{~m}$. As unidades básicas (UB) foram compostas por uma planta as quais resultaram em 24 UB linha $^{-1}$. Foi utilizada estufa plástica no terceiro experimento, com dimensões de $24 \mathrm{~m}$ de comprimento por
$10 \mathrm{~m}$ de largura com área útil de 9,6 $\mathrm{m}$ de largura e 19,2 m de comprimento, com pé-direito de $2 \mathrm{~m}$ e de $3,5 \mathrm{~m}$ na parte central. Toda a cobertura da estufa, bem como as cortinas móveis das laterais e das portas, consistiram em filme de polietileno de baixa densidade (PEBD), com espessura de 100 micras e aditivo anti-UV. Nas duas extremidades Norte e Sul, localizavam-se as portas, cada uma com dimensão de 2,0 x 2,0 m. A unidade básica (UB) foi composta por uma planta e resultou em 24 unidades básicas, em cada uma das oito fileiras com o mesmo espaçamento dos túneis.

As plantas foram transplantadas quando as mudas apresentaram quatro folhas definitivas ou $10 \mathrm{~cm}$ de estatura. Todos os tratos culturais foram realizados com base na recomendação para a cultura (Filgueira, 2000), sendo aplicados concomitantemente nos três experimentos. Os frutos de tomate foram colhidos quando surgiu pigmentação diferenciada vermelha e foram colocados em sacos de papel e identificados, para posterior determinação da fitomassa fresca dos frutos, usando uma balança digital com precisão de um grama.

Cada planta foi considerada como uma unidade básica (UB). A seqüência de duas, três e quatro plantas na linha formou as parcelas de duas, três e quatro UB de tamanho (X). O número de repetições de cada tamanho de parcela $(\mathrm{X})$ ficou limitado $\mathrm{N} / \mathrm{X}$, sendo $\mathrm{N}$ o número total de plantas do experimento. Nos experimentos instalados nos túneis, as culturas foram compostas por 72 plantas, nesse caso, 72 unidades básicas (UB), $(\mathrm{N}=72$ o número total de plantas dos experimentos). Dessa forma, para $\mathrm{X}=1,2,3$ e 4 , o número de parcelas foi, respectivamente, de 72, 36, 24 e 18 parcelas. Já o experimento realizado na estufa foi composto por 192 plantas, nesse caso $192 \mathrm{UB}$, formando para $\mathrm{X}=$ 1, 2, 3 e 4, 192, 96, 64 e 48 parcelas, respectivamente.

Foram realizadas análises considerando apenas as colheitas individuais e também as colheitas acumuladas, em cada caso simulado. Para tanto os grupos formados seguiram a seqüência da divisão das colheitas obtidas pelos seus múltiplos nos diferentes experimentos. Assim, as múltiplas colheitas sucessi- 
vas foram agrupadas: a) agrupamento duas a duas $\left(1^{\mathrm{a}}+2^{\mathrm{a}} ; 3^{\mathrm{a}}+4^{\mathrm{a}} ; \ldots ; 11^{\mathrm{a}}+12^{\mathrm{a}}\right)$; b) agrupamento três a três $\left(1^{\mathrm{a}}+2^{\mathrm{a}}+3^{\mathrm{a}}\right.$; $\left.4^{\mathrm{a}}+5^{\mathrm{a}}+6^{\mathrm{a}} ; \ldots ; 10^{\mathrm{a}}+11^{\mathrm{a}}+12^{\mathrm{a}}\right) ;$ c) agrupamento quatro a quatro $\left(1^{\mathrm{a}}+2^{\mathrm{a}}+3^{\mathrm{a}}+4^{\mathrm{a}}\right.$; $\left.\ldots ; 9^{\mathrm{a}}+10^{\mathrm{a}}+11^{\mathrm{a}}+12^{\mathrm{a}}\right)$ e d) agrupamento seis a seis $\left(1^{\mathrm{a}}+2^{\mathrm{a}}+3^{\mathrm{a}}+4^{\mathrm{a}}+5^{\mathrm{a}}+6^{\mathrm{a}}\right.$; $\left.7^{\mathrm{a}}+8^{\mathrm{a}}+9^{\mathrm{a}}+10^{\mathrm{a}}+11^{\mathrm{a}}+12^{\mathrm{a}}\right)$.

Para cada uma das colheitas individuais, colheitas agrupadas e tamanhos de parcelas foram estimadas $\mathrm{M}(\mathrm{x})=$ média das parcelas com X UB de tamanho, $\mathrm{V}(\mathrm{x})=$ variância entre as parcelas de $\mathrm{X}$ unidades básicas de tamanho, V1= variância entre as parcelas de uma unidade básica de tamanho, $\mathrm{VU}(\mathrm{X})=\mathrm{V}(\mathrm{x}) / \mathrm{X}^{2}$ variância por UB entre as parcelas de $X$ $\mathrm{UB}, \mathrm{VR}(\mathrm{X})=\mathrm{V}(\mathrm{x}) / \mathrm{X}$, variância reduzida e $\mathrm{CV}(\mathrm{x})=$ coeficiente de variação entre as parcelas de $\mathrm{X}$ unidades básicas de tamanho. A partir dessas estimativas, foi calculado o índice de informação relativa (IR), pelo método da máxima informação relativa, atribuindo $100 \%$ às parcelas cujo tamanho fosse igual a uma UB. Para os demais tamanhos de parcela simulados, as IR foram obtidas pela expressão $\mathrm{IR}(\mathrm{x})=100 * \mathrm{~V} 1 / \mathrm{VR}(\mathrm{x})$ (Keller, 1949). Este método objetiva extrair a maior quantidade de informação numa unidade de área assim como avaliar a melhor forma para uma parcela de determinado tamanho. Ocorre o decréscimo da IR com o aumento do tamanho, sendo o tamanho ideal para determinado experimento aquele a partir do qual há uma estabilidade nos decréscimos.

Em cada experimento realizado aplicou-se o teste de Bartlett (Steel et al., 1997), entre as variâncias das colheitas individuais e agrupadas em cada tamanho de parcela simulado, para verificar a homogeneidade entre as variâncias dentro de cada colheita em diferentes tamanhos de parcela. Em todas as análises estatísticas foi adotado $5 \%$ de probabilidade de erro.

\section{RESULTADOS E DISCUSSÃO}

A frequência média entre as colheitas foi de 3,5 dias, totalizando um período de 42 dias de colheita. Nesse sentido Cargnelutti Filho et al. (2004), estudando a repetibilidade da produção de genótipos de tomateiro, orientam que sejam colhidos no mínimo sete vezes para que se possa obter uma boa discriminação da produção. No entanto, Lopes et al. (1998), avaliando o comportamento do percentual de frutos colhidos de tomate em cada colheita e sua influência nos valores do teste de Tukey, recomenda que se utilize apenas o terço inicial de colheitas. O percentual médio de plantas colhidas na estufa e nos túneis 1 e 2 foi de 70,66, 63,08 e $63,43 \%$, respectivamente.

Com o aumento do número de plantas por parcela e o agrupamento de colheitas, foram verificados acréscimos nos valores de variâncias (Tabela 1). Esse comportamento deve-se ao fato de estas combinações apresentarem valores de médias consideravelmente superiores aos de colheitas individuais ou de uma planta por parcela. No início do ciclo de produção as estimativas das variâncias foram menores e, com o passar das colheitas realizadas, foram verificados acréscimos naquelas, principalmente a partir da quinta colheita, chegando a um máximo na sétima e oitava colheitas para estufas e túneis, respectivamente, estando relacionado ao fato de ser nessas colheitas que se concentram os maiores valores médios de produção da cultura. Estes resultados concordam com Fayad et al. (2001) que estudando a produção e a classificação de tomate em cultivo protegido, mostraram que na metade e na parte final do ciclo de cultivo concentram-se as maiores produções de frutos.

A partir da oitava colheita houve um decréscimo nas estimativas da variância, explicado pelo fato das colheitas apresentarem redução da produção, com a proximidade do final do ciclo. Assim a diferença entre as produções das unidades básicas reduz, influenciando diretamente as estimativas das variâncias. Ao final do ciclo produtivo a variabilidade aumenta novamente em decorrência dos aspectos fisiológicos das plantas, pois estas passam para o estado de senescência.

A partir das estimativas das variâncias reduzidas a uma unidade básica entre parcelas de diferentes tamanhos, Vallejo \& Mendonza (1992) aplicaram sucessivos testes de homogeneidade de variâncias até encontrarem um grupo de tamanhos de parcela tal que não apre- sentasse heterogeneidade das variâncias, sendo escolhido o menor tamanho desse grupo. Desta forma o agrupamento de seis colheitas para tamanhos de parcela iguais ou maiores a duas plantas foi a simulação que apresentou o melhor resultado.

Independentemente do tamanho de parcela, com a evolução das colheitas, ocorreu decréscimo do coeficiente de variação (Tabela 2), resultando em ganho de precisão experimental. Isto acontece porque o aumento do valor do desvio padrão nas colheitas se dá em uma taxa inferior ao incremento da média. Assim, observa-se redução na magnitude deste indicador de precisão, devido principalmente ao preenchimento das lacunas formadas pelas plantas não colhidas (produção nula na colheita), o que resultou no aumento da média. Esse aumento da média nas colheitas também é intimamente relacionado com o número de plantas colhidas.

Entretanto o coeficiente de variação decresceu quando se acumulou a primeira colheita com a segunda, porém em taxas menores com o acúmulo das colheitas subseqüentes. Essa redução ocorreu, principalmente, devido ao aumento da média propiciada pelas plantas que antes não haviam sido colhidas, reduzindo assim as produções nulas. Essa constatação indica que os experimentos devem conter polo menos três colheitas durante o período produtivo.

Os valores de $\mathrm{CV}$ na fase inicial de colheitas maiores concordam com Lana et al. (1953), que obtiveram valores maiores de variabilidade nas colheitas iniciais. Cargnelutti Filho et al. (2004) encontraram valores de $\mathrm{CV}$ maiores no início e final das colheitas de tomate, atribuídos ao fato de, nestes estágios de desenvolvimento, o início e o término na emissão de frutos não serem uniformes, e, por conseqüência, alternarem as produções. Ainda segundo esses autores, são as colheitas intermediárias que apresentam picos de produção em qualidade e quantidade, e os dados de CV são mais baixos, resultando em uma maior precisão experimental.

Os túneis mostraram um CV maior em relação à estufa nas colheitas individuais nos diferentes tamanhos de parcela (Tabela 2). A medida em que se aumen- 
Tabela 1.Variâncias da fitomassa de frutos $\left(\mathrm{g}^{2} \times 10^{3}\right)$ entre colheitas e entre os tamanhos de parcela simulados para tomate cultivado em ambiente protegido (variance of fruits fitomass $\left(\mathrm{g}^{2} \times 10^{3}\right)$ among crops and the plot sizes simulated in the same crop, for tomato cultivated in greenhouse). Santa Maria, UFSM, 2007.

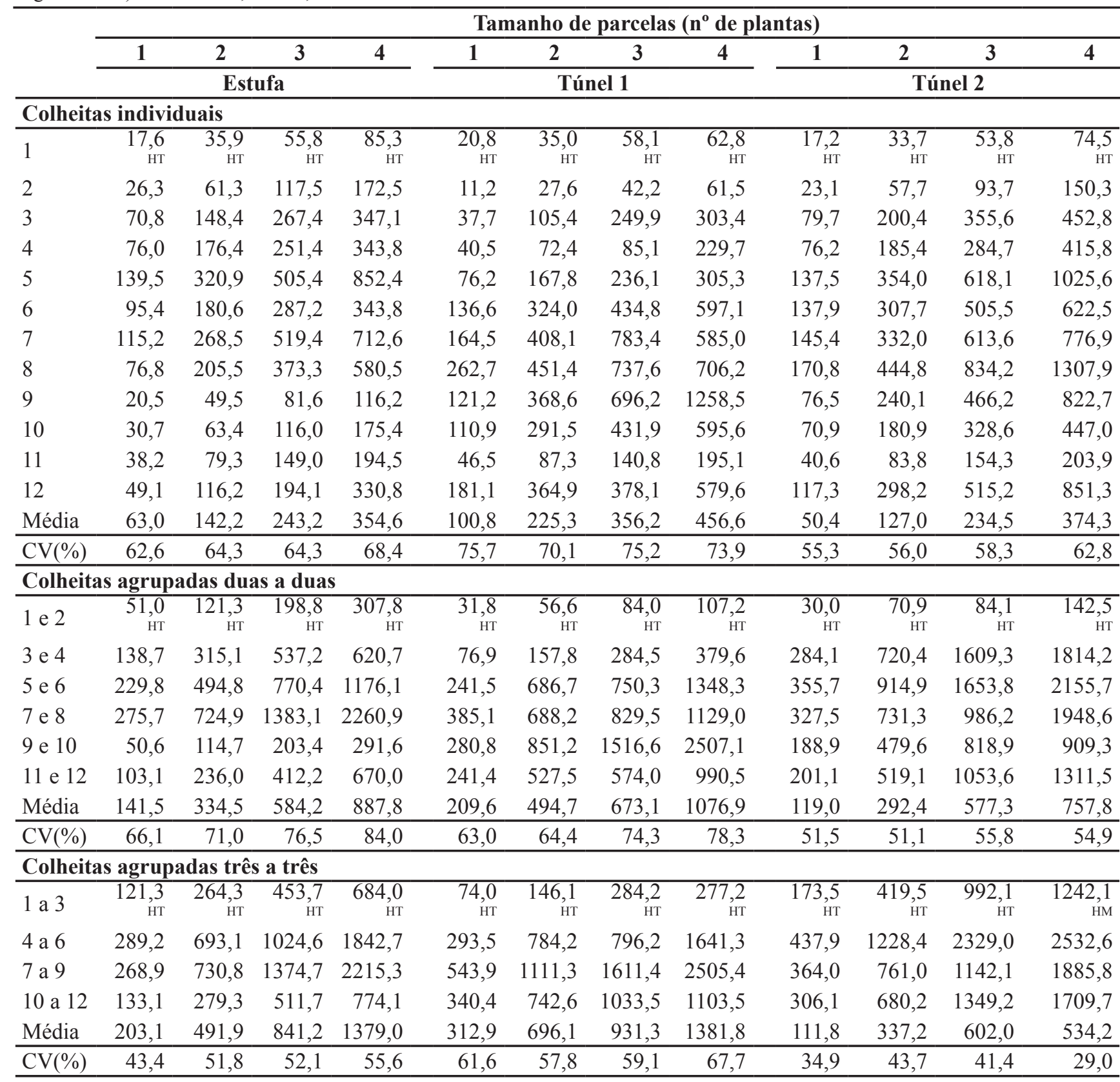

\section{Colheitas agrupadas quatro a quatro}

\begin{tabular}{|c|c|c|c|c|c|c|c|c|c|c|c|c|}
\hline 1 a 4 & $\begin{array}{r}177,7 \\
\text { HT }\end{array}$ & $\begin{array}{r}453,9 \\
\text { HT }\end{array}$ & $\begin{array}{r}819,7 \\
\text { HT }\end{array}$ & $\begin{array}{r}1100,9 \\
\text { HT }\end{array}$ & $\begin{array}{r}113,6 \\
\text { HT }\end{array}$ & $\begin{array}{r}214,1 \\
\text { HT }\end{array}$ & $\begin{array}{r}359,5 \\
\text { HT }\end{array}$ & $\begin{array}{r}466,6 \\
\text { HT }\end{array}$ & $\begin{array}{r}328,5 \\
\text { HT }\end{array}$ & $\begin{array}{r}827,7 \\
\text { HT }\end{array}$ & $\begin{array}{r}1937,8 \\
\mathrm{HM}\end{array}$ & $\begin{array}{r}2404,1 \\
\text { HT }\end{array}$ \\
\hline 5 a 8 & 426,0 & 1044,8 & 1787,0 & 2964,3 & 699,3 & 1463,8 & 1257,4 & 2192,9 & 636,0 & 1547,8 & 2775,5 & 4274,9 \\
\hline 9 a 12 & 156,3 & 335,7 & 605,9 & 878,3 & 489,3 & 1219,0 & 1997,7 & 2515,6 & 339,7 & 711,4 & 1501,5 & 1685,5 \\
\hline Média & 253,3 & 611,5 & 1070,9 & 1647,8 & 434,1 & 965,6 & 1204,9 & 1725,0 & 174,4 & 453,1 & 647,5 & 1336,8 \\
\hline $\mathrm{CV}(\%)$ & 59,2 & 62,1 & 58,8 & 69,5 & 68,4 & 68,6 & 68,1 & 63,9 & 40,1 & 44,0 & 31,3 & 47,9 \\
\hline \multicolumn{13}{|c|}{ Colheitas agrupadas seis a seis } \\
\hline 1 a 6 & $\begin{array}{r}394,0 \\
\text { HM }\end{array}$ & $\begin{array}{r}965,1 \\
\mathrm{HM}\end{array}$ & $\begin{array}{r}1613,1 \\
\text { HM }\end{array}$ & $\begin{array}{r}2782,0 \\
\text { HM }\end{array}$ & $\begin{array}{r}425,9 \\
\text { H }\end{array}$ & $\begin{array}{r}1160,5 \\
\mathrm{HM}\end{array}$ & $\begin{array}{r}1347,2 \\
\text { HT }\end{array}$ & $\begin{array}{r}2625,5 \\
\text { HM }\end{array}$ & $\begin{array}{r}687,5 \\
\mathrm{HM}\end{array}$ & $\begin{array}{r}1833,9 \\
\mathrm{HM}\end{array}$ & $\begin{array}{r}3878,4 \\
\mathrm{HM}\end{array}$ & $\begin{array}{r}4591,5 \\
\mathrm{HM}\end{array}$ \\
\hline 7 a 12 & 462,3 & 1281,3 & 2505,4 & 3665,4 & 978,4 & 2004,7 & 3227,4 & 4012,7 & 602,5 & 1386,9 & 2280,1 & 2875,9 \\
\hline Média & 428,1 & 1123,2 & 2059,2 & 3223,7 & 702,2 & 1582,6 & 2287,3 & 3319,1 & 60,1 & 316,1 & 1130,2 & 1213,1 \\
\hline $\mathrm{CV}(\%)$ & 11,3 & 19,9 & 30,6 & 19,4 & 55,6 & 37,7 & 58,1 & 29,6 & 9,3 & 19,6 & 36,7 & 32,5 \\
\hline
\end{tabular}

*HM: Variâncias homogêneas entre colheitas; (homogeneous variances among crops); HT: Variâncias heterogêneas entre colheitas pelo teste de Bartlett em nível de 5\% de probabilidade de erro (heterogeneous variances among crops or the Bartlett test to 5\% of error probability). 
Tabela 2. Coeficiente de variação (\%) da fitomassa de frutos entre colheitas e entre os tamanhos de parcela simulados na mesma colheita, para tomate cultivado em ambiente protegido (coefficient of variation (\%) of the fruits fitomass among crops and among simulated plot sizes in the same crop, for tomato cultivated in greenhouse) Santa Maria, UFSM, 2007.

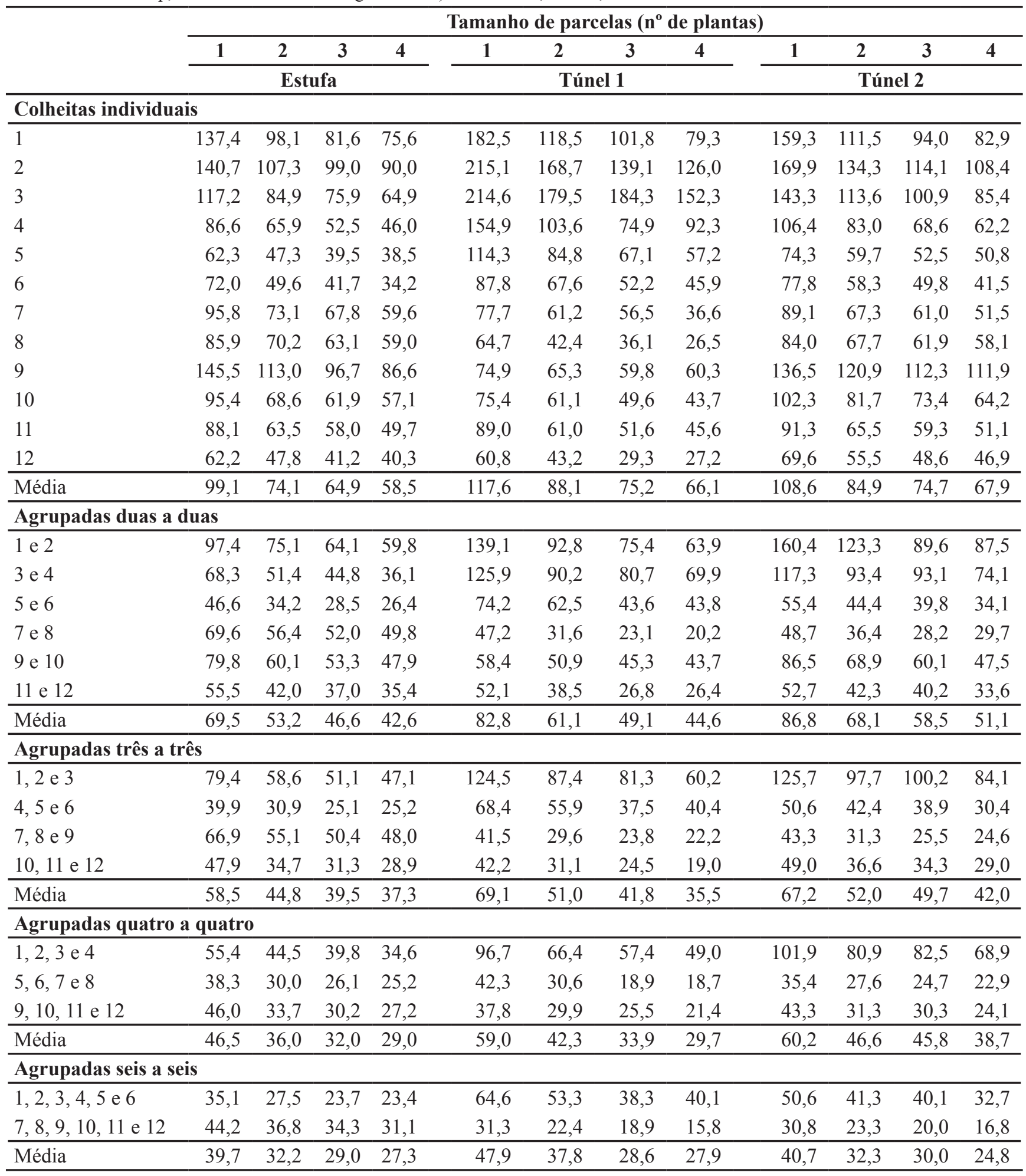

taram os tamanhos dos agrupamentos e os de parcela, o CV praticamente estabilizou, especialmente no agrupamento de seis colheitas nos tamanhos de parcela três e quatro plantas para estufa e túneis. Desta forma, a utilização de mais plantas por parcela, além de favorecer a redução dos valores de $\mathrm{CV}$, permite uma melhor distribuição dos valores ao longo das sucessivas colheitas.

As reduções dos valores do índice de informação relativa (IR) foram graduais com o aumento de parcela (Tabela 3) influenciado principalmente pelo aumento da variância quando simuladas parcelas com mais plantas. À medida que se aumentou o número de colheitas por agrupamento este comportamento 
Tabela 3. Índice de informação relativa (\%) da fitomassa de frutos entre colheitas e entre os tamanhos de parcela simulados na mesma colheita, para tomate cultivado em ambiente protegido (index of relative information (\%) of the fruits fitomass among crops and among the plot sizes simulated in the same crop, for tomato cultivated in greenhouse). Santa Maria, UFSM. 2007.

\begin{tabular}{|c|c|c|c|c|c|c|c|c|c|}
\hline & \multicolumn{9}{|c|}{ Tamanho de parcelas ( $n^{0}$ de plantas) } \\
\hline & 2 & 3 & 4 & 2 & 3 & 4 & 2 & 3 & 4 \\
\hline & \multicolumn{3}{|c|}{ Estufa } & \multicolumn{3}{|c|}{ Túnel 1} & \multicolumn{3}{|c|}{ Túnel 2} \\
\hline \multicolumn{10}{|c|}{ Colheitas individuais } \\
\hline 1 & 98,1 & 94,6 & 82,5 & 118,7 & 107,2 & 132,5 & 102,0 & 95,8 & 92,3 \\
\hline 2 & 85,9 & 67,3 & 61,1 & 81,3 & 79,7 & 72,8 & 80,0 & 73,9 & 61,4 \\
\hline 3 & 95,4 & 79,4 & 81,6 & 71,5 & 45,2 & 49,6 & 79,5 & 67,2 & 70,4 \\
\hline 4 & 86,2 & 90,7 & 88,5 & 111,8 & 142,7 & 70,5 & 82,1 & 80,3 & 73,3 \\
\hline 5 & 87,0 & 82,8 & 65,5 & 90,8 & 96,8 & 99,8 & 77,7 & 66,7 & 53,6 \\
\hline 6 & 105,7 & 99,7 & 111,1 & 84,3 & 94,2 & 91,5 & 89,6 & 81,8 & 88,6 \\
\hline 7 & 85,8 & 66,6 & 64,7 & 80,6 & 63,0 & 112,5 & 87,6 & 71,1 & 74,9 \\
\hline 8 & 74,8 & 61,7 & 52,9 & 116,4 & 106,9 & 148,8 & 76,8 & 61,4 & 52,2 \\
\hline 9 & 82,8 & 75,4 & 70,6 & 65,8 & 52,2 & 38,5 & 63,7 & 49,2 & 37,2 \\
\hline 10 & 96,8 & 79,3 & 69,9 & 76,1 & 77,0 & 74,5 & 78,4 & 64,7 & 63,4 \\
\hline 11 & 96,4 & 76,9 & 78,5 & 106,7 & 99,2 & 95,4 & 96,9 & 79,0 & 79,7 \\
\hline 12 & 84,5 & 75,9 & 59,4 & 99,2 & 143,7 & 125,0 & 78,7 & 68,3 & 55,1 \\
\hline Média & 89,9 & 79,2 & 73,9 & 91,9 & 92,3 & 92,6 & 82,8 & 71,6 & 66,8 \\
\hline CV (\%) & 9,5 & 14,4 & 21,4 & 19,8 & 33,9 & 36,0 & 12,2 & 16,5 & 24,1 \\
\hline \multicolumn{10}{|c|}{ Agrupadas duas a duas } \\
\hline 1 e 2 & 84,2 & 77,1 & 66,3 & 112,4 & 113,5 & 118,6 & 84,6 & 106,9 & 84,1 \\
\hline 3 e 4 & 88,0 & 77,5 & 89,4 & 97,5 & 81,1 & 81,1 & 78,9 & 53,0 & 62,6 \\
\hline 5 e 6 & 92,9 & 89,5 & 78,2 & 70,3 & 96,6 & 71,6 & 77,8 & 64,5 & 66,0 \\
\hline 7 e 8 & 76,1 & 59,8 & 48,8 & 111,9 & 139,3 & 136,4 & 89,6 & 99,6 & 67,2 \\
\hline 9 e 10 & 88,3 & 74,7 & 69,5 & 66,0 & 55,6 & 44,8 & 78,8 & 69,2 & 83,1 \\
\hline 11 e 12 & 87,4 & 75,1 & 61,6 & 91,5 & 126,1 & 97,5 & 77,5 & 57,3 & 61,3 \\
\hline Média & 86,1 & 75,6 & 69,0 & 91,6 & 102,0 & 91,7 & 81,2 & 75,1 & 70,7 \\
\hline CV $(\%)$ & 6,6 & 12,5 & 20,2 & 21,8 & 30,1 & 36,1 & 6,0 & 30,2 & 14,4 \\
\hline \multicolumn{10}{|c|}{ Agrupadas três a três } \\
\hline 1,2 e 3 & 91,8 & 80,2 & 71,0 & 101,3 & 78,1 & 106,8 & 82,7 & 52,5 & 55,9 \\
\hline 4,5 e 6 & 83,4 & 84,7 & 62,8 & 74,8 & 110,6 & 71,5 & 71,3 & 56,4 & 69,2 \\
\hline 7,8 e 9 & 73,6 & 58,7 & 48,6 & 97,9 & 101,3 & 86,8 & 95,7 & 95,6 & 77,2 \\
\hline 10,11 e 12 & 95,3 & 78,0 & 68,8 & 91,7 & 98,8 & 123,4 & 90,0 & 68,1 & 71,6 \\
\hline Média & 86,0 & 75,4 & 62,8 & 91,4 & 97,2 & 97,1 & 84,9 & 68,1 & 68,5 \\
\hline CV $(\%)$ & 11,2 & 15,2 & 16,1 & 12,9 & 14,1 & 23,4 & 12,4 & 28,6 & 13,2 \\
\hline \multicolumn{10}{|c|}{ Agrupadas quatro a quatro } \\
\hline $1,2,3$ e 4 & 78,3 & 65,0 & 64,6 & 106,2 & 94,8 & 97,4 & 79,4 & 50,9 & 54,7 \\
\hline $5,6,7$ e 8 & 81,5 & 71,5 & 57,5 & 95,5 & 166,8 & 127,6 & 82,2 & 68,7 & 59,5 \\
\hline $9,10,11$ e 12 & 93,1 & 77,4 & 71,2 & 80,3 & 73,5 & 77,8 & 95,5 & 67,9 & 80,6 \\
\hline Média & 84,3 & 71,3 & 64,4 & 94,0 & 111,7 & 100,9 & 85,7 & 62,5 & 64,9 \\
\hline $\mathrm{CV}(\%)$ & 9,3 & 8,7 & 10,6 & 13,9 & 43,8 & 24,8 & 10,0 & 16,1 & 21,3 \\
\hline \multicolumn{10}{|c|}{ Agrupadas seis a seis } \\
\hline $1,2,3,4,5$ e 6 & 81,6 & 73,3 & 56,6 & 73,4 & 94,8 & 64,9 & 75,0 & 53,2 & 59,9 \\
\hline $7,8,9,10,11$ e 12 & 72,2 & 55,4 & 50,4 & 97,6 & 90,9 & 97,5 & 86,9 & 79,3 & 83,8 \\
\hline Média & 76,9 & 64,3 & 53,6 & 85,5 & 92,9 & 81,2 & 80,9 & 66,2 & 71,8 \\
\hline CV (\%) & 8,7 & 19,7 & 8,2 & 20,0 & 2,9 & 28,4 & 10,4 & 27,9 & 23,5 \\
\hline
\end{tabular}


também foi verificado, mostrando que os valores das variâncias por unidade básica aumentaram em maior proporção em parcelas maiores.

Na estufa, os agrupamentos de duas, três e quatro colheitas mostram que não ocorreram reduções consideráveis para o IR, mas uma redução foi verificada quando do agrupamento de seis colheitas. Também, as maiores reduções neste índice foram devido ao aumento do tamanho de parcela quando comparado com o aumento do agrupamento (Tabela 3 ). Keller (1949) constatou que o IR decresce com o aumento do tamanho da parcela, sendo o tamanho ideal aquele a partir do qual há uma estabilidade nos decréscimos. $\mathrm{O}$ autor comenta ainda que, com o aumento da variância comparável à informação relativa da parcela, esta tende a decrescer levando geralmente a recomendações de uso de parcelas de uma unidade básica.

Desta forma pode-se inferir que os agrupamentos das colheitas de seis em seis colheitas com parcelas de quatro plantas, proporcionaram as melhores condições de baixo CV e IR próximos a $100 \%$, independente do ambiente de cultivo.

As variâncias, entre as colheitas agrupadas, são homogêneas a partir do agrupamento de seis colheitas. Há acréscimos nas estimativas da variância da fitomassa de frutos com o aumento do tamanho da parcela e/ou o número de colheitas agrupadas.

Parcelas de quatro plantas na linha de cultivo, combinados com agrupamento de seis em seis colheitas reduzem a variabilidade.

\section{AGRADECIMENTOS}

Ao CNPq e CAPES pelo auxílio financeiro para a realização do trabalho e pela concessão de bolsa de produtivida- de em pesquisa aos autores Alessandro Dal'Col Lúcio e Lindolfo Storck.

\section{REFERÊNCIAS}

CAMARGO FILHO WP; CAMARGO FP. 2008. Planejamento da produção sustentável de hortaliças folhosas: organização das informações decisórias ao cultivo. Informações Econômica 38: 27-36.

CARGNELUTTI FILHO A; RADIN B; MATZENAUER R; STORCK L. 2004. Número de colheitas e comparação de genótipos de tomateiro cultivados em estufa de plástico. Pesquisa Agropecuária Brasileira 39: 953-959.

CARPES RH; LÚCIO AD; STORCK L; LOPES SJ; ZANARDO B; PALUDO AL. 2008. Ausência de frutos colhidos e suas interferências nas estimativas da fitomassa de frutos de abobrinha italiana cultivada em diferentes sistemas de irrigação. Revista Ceres 55: 590-595.

CERMEÑO ZS. 1990. Estufas - instalações e manejo. Lisboa: Litexa. 355p.

EMBRAPA. 1999. Sistema brasileiro de classificação de solos. Brasília: EMBRAPA. 412p.

FAYAD JA; FONTES PCR; CARDOSO AA; FINGER FL. 2001. Crescimento e produção do tomateiro cultivado sob condições de campo e de estufa. Horticultura Brasileira 19: 232-237.

FEIJÓ S; STORCK L; LÚCIO AD; LOPES SJ; GARCIA DC; CARPES RH. 2008. Heterogeneity index of zucchini yield on a protected environment and experimental planning. Horticultura Brasileira 26: 35-39.

FILGUEIRA FAR. 2000. Novo manual de olericultura. Viçosa: UFV. 402p.

IBGE - Instituto Brasileiro de Geografia e Estatística. 2008, 20 de março. Produção Agrícola Municipal (PAM), Rio de Janeiro. Disponível em http://www.sidra.ibge.gov.br.

KELLER K. 1949. Uniformity trials on hops, Humulus lupulus L., for increasing the precision of field experiments. Agronomy Journal 41:389-392.

LANA EP; HOMEYER PG; HABER ES. 1953. Field plot technique in vegetable crops. American Society for Horticultural Science 78: 20-30.

LOPES SJ; STORCK L; HELDWEIN AB; FEIJÓ S; ROS CA. 1998. Técnicas experimentais para tomateiro tipo salada sob estufas plásticas. Ciência Rural 28:193-197.

LORENTZ LH; LÚCIO AD; STORCK L; LOPES
SJ; BOLIGON AA; CARPES RH. 2004. Variação temporal do tamanho de amostra para experimentos em estufa plástica. Ciência Rural 34: 1043-1049.

LORENTZ LH; LÚCIO AD; BOLIGON AA; LOPES SJ; STORCK L. 2005. Variabilidade da produção de frutos de pimentão em estufa plástica. Ciência Rural 35: 316-323.

LÚCIO AD; CARPES RH; STORCK L; LOPES SJ; LORENTZ LH; PALUDO AL. 2008. Variância e média da massa de frutos de abobrinha-italiana em múltiplas colheitas. Horticultura Brasileira 26: 335-341.

LÚCIO AD; LORENTZ LH; BOLIGON AA; LOPES SJ; STORCK L; CARPES RH. 2006. Variação temporal da produção de pimentão influenciada pela posição e características morfológicas das plantas em ambiente protegido. Horticultura Brasileira 24: 31-35.

LÚCIO AD; MELLO RM; STORCK L; CARPES RH; BOLIGON AA; ZANARDO B. 2004. Estimativa de parâmetros para o planejamento de experimentos com a cultura do pimentão em área restrita. Horticultura Brasileira 22: 66-770.

LÚCIO AD; SOUZA MF; HELDWEIN AB; LIEBERKNECHT D; CARPES RH; CARVALHO MP. 2003. Tamanho da amostra e método de amostragem para avaliação de características do pimentão em estufa plástica. Horticultura Brasileira 21:180-184.

MARODIM VS; STORCK L; LOPES SJ. 2000. Delineamento experimental e tamanho de amostra para alface cultivada em hidroponia. Ciência Rural 30: 779-781.

MELLORM; LÚCIOAD; STORCK L; LORENTZ LH; CARPES RH; BOLIGON AA. 2004. Size and form of plots for the culture of the italian pumpkin in plastic greenhouse. Scientia Agricola 61: 457-461.

SOUZA MF; LÚCIO AD; STORCK L; CARPES RH; SANTOS PM; SIQUEIRA LF. 2002. Tamanho da amostra para peso da massa de frutos, na cultura da abóbora italiana em estufa plástica. Revista Brasileira de Agrociência 8: 131-136.

STEEL RGD; TORRIE JH; DICKEY DA. 1997. Principles and procedures of statistics: a biometrical approach. 3ed. New York: McGraw-Hill, 666p.

STORCK L; GARCIA DC; LOPES SJ; ESTEFANEL V. 2006. Experimentação vegetal. Santa Maria: UFSM, 198p.

VALLEJO RL; MENDONZA HA. 1992. Plot technique studies on sweet potato yield trials. Journal American Society Science 117: 508-511. 